\title{
Optimizing separation conditions for polycyclic aromatic hydrocarbons in micellar electrokinetic chromatography
}

\author{
Ming-Mu Hsieh, Yui-Chun Kuo, Pei-Ling Tsai, Huan-Tsung Chang* \\ Department of Chemistry, National Taiwan University, P.O. Box 23-34, 1 Roosevelt Road Section 4, Taipei 10764, Taiwan
}

\begin{abstract}
We report the separation of polycyclic aromatic hydrocarbons (PAHs) using $0.1 \%$ poly(ethylene oxide) (PEO) in micellar electrokinetic chromatography (MEKC). In the presence of PEO, adsorption of PAHs on the capillary wall was reduced, leading to better resolution and reproducibility. Effects of tetrapentylammonium iodide (TPAI), dextran sulfate (DS), methanol, and sodium lauryl sulfate (SDS) on the separation of PAHs were elucidated. In terms of resolution and speed, DS, compared to TPAI, is a better additive for separation of PAHs. When using $0.1 \%$ PEO solution containing $45 \%$ methanol, 50 $\mathrm{m} M$ SDS, and $0.02 \%$ DS, separation of 10 PAHs containing 2 to 5 benzene rings was accomplished in less than 12 min at 15 $\mathrm{kV}$ in a commercial CE system. The method has also been tested for separating seven PAHs with high quantum yields when excited at $325 \mathrm{~nm}$ using a He-Cd laser. Unfortunately, separation of the seven PAHs was not achieved and sensitivity diminished under the same conditions. To optimize sensitivity, resolution and speed, a stepwise technique in MEKC has been proposed. The seven PAHs were resolved in $35 \mathrm{~min}$ at $15 \mathrm{kV}$ when separation was performed in $0.1 \%$ PEO solution containing $35 \mathrm{~m} M$ SDS, $40 \%$ methanol and $0.02 \%$ DS for $2 \mathrm{~min}$, and subsequently in $0.1 \%$ PEO solution containing $20 \mathrm{~m} M$ SDS, 50\% methanol, and 0.02\% DS. (C) 2001 Elsevier Science B.V. All rights reserved.
\end{abstract}

Keywords: Background electrolyte composition; Polynuclear aromatic hydrocarbons

\section{Introduction}

Micellar electrokinetic chromatography (MEKC) has been frequently chosen for separations of charged or neutral compounds, such as phenol and hydrophobic hydrocarbons, based on their relative affinity for the lipophilic interior and/or the ionic exterior of a micellar pseudostationary phase [1-5]. Although MEKC is very promising for separation of neutral compounds, a narrow separation window and a limited stability of micellar pseudostationary phases are problematic.

Due to the abundance in the environment and the

*Corresponding author. Tel./fax: +886-2-2362-1963.

E-mail address: changht@mail.ch.ntu.edu.tw (H.-T. Chang). adverse health effects to which they have been linked, the analysis of polycyclic aromatic hydrocarbons (PAHs) is of interest and importance [6]. MEKC is one of the most important techniques for this purpose [7-9]. It however is difficult to achieve any degree of selectivity using micelles alone because binding to the micelle is so strong. Irreproducibility and loss of resolution due to adsorption of PAHs on the capillary wall is also a common problem [10,11]. As concentrations and types of detergents, organic solvents, and other additives are important determinants for the capacity factors $\left(k^{\prime}\right)$ of PAHs, controlling these factors is typical to solve those problems in MEKC [12].

The separation of PAHs by MEKC has been successfully achieved using micelles, such as sodium 
lauryl sulfate (SDS) micelles, along with modifiers such as cyclodextrins [13], urea [14], and organic solvents [15]. The use of organic solvent is generally effective to improve solubility, to optimize capacity factors of PAHs, to control electroosmotic flow (EOF), and to minimize adsorption of PAHs on the capillary wall. For example, selectivity is improved using micelles commonly prepared in 40 to $60 \%$ acetonitrile or methanol [16]. Alternatively, selectivity for separating PAHs can also be improved by carefully choosing hydrophobic alkylammonium ions because the magnitude of the alteration of the migration mobilities of PAHs is related to the chain length, structure, and concentration of ammonium ions [17]. Problems associated with the use of ammonium ions include changes in EOF and poor reproducibility as a result of adsorption on the capillary wall. Planar organic cations that form charge-transfer complexation with PAHs have been used for separation of PAHs in nonaqueous capillary electrophoresis (CE) [18]. Recently, separation of PAHs in monomolecular pseudostationary phase formed from ionic polymers has been demonstrated in MEKC [19-21]. Advantages of the alternative over conventional micelles include its stability in organic phase and providing a wider separation window. Capillary electrochromatography (CEC) is also useful for separation of PAHs [22-24]. In CEC, PAHs migrated through stationary phase by EOF, while, like in high-performance liquid chromatography, the separation is determined by its partition between stationary phase and mobile phase. Although reasonable resolution is obtained, polymerization of small particles inside capillaries or packing the small particles into small diameter of capillaries is not so easy.

Poor sensitivity is one of problems commonly associated with analysis of PAHs in environmental and biological samples via MEKC with absorbance detection. To increase sensitivity, laser-induced fluorescence (LIF) using UV lasers has been developed [25-27]. By taking the advantage of high sensitivity with LIF, this paper is devoted to provide a new approach for analysis of PAHs in MEKC with high resolving power, speed, and sensitivity. Effects of SDS pseudostationary phase, methanol, tetrapentylammonium iodide (TPAI), dextran sulfate (DS), and poly(ethylene oxide) (PEO) on resolution and speed were carefully evaluated. We also proposed a stepwise technique based on changes in concentration of SDS and methanol using a $\mathrm{He}-\mathrm{Cd}$ laser for optimum resolution, speed and sensitivity.

\section{Experimental}

\subsection{Apparatus}

A commercial electrophoresis instrument from Bio-Rad (BioFocus CE 2000, Hercules, CA, USA) was used for analysis of PAHs with absorbance detection. The fused-silica capillaries (Polymicro Technologies, Phoenix, AZ, USA) were $38 \mathrm{~cm} \times 75$ $\mu \mathrm{m}$ I.D. At $33 \mathrm{~cm}$ from the injection end, the polyimide coating was burned off to form the detection window. The absorbance was obtained at $254 \mathrm{~nm}$.

The basic design for analysis of PAHs in MEKC with LIF has been previously described [25]. Briefly, a high-voltage power supply (Gamma High Voltage Research, Ormond Beach, FL, USA) was used to drive electrophoresis. The entire detection system was enclosed in a black box with a high-voltage interlock. High-voltage end of the separation system was put in a laboratory-made Plexiglass box for safety. A 35-mW He-Cd laser with 325-nm output from Melles Griot (Carlsbad, CA, USA) was used for excitation. The emission light was collected with a $10 \times$ objective (numerical aperture $=0.25$ ). One cutoff filter (GG 395) was used to block scattered light before the emitted light reached the phototube (Hamamatsu R928). The amplified currents were transferred directly through a $10-\mathrm{k} \Omega$ resistor to a 24-bit A/D interface (Borwin, JMBS Developments, Le Fontanil, France) at $10 \mathrm{~Hz}$ and stored in a personal computer.

\subsection{Materials}

All PAHs and TPAI were of reagent grade and were obtained from Acros (Pittsburgh, PA, USA). Sodium hydroxide was from Fisher (Fair Lawn, NJ, USA). Dextran sulfate [molecular mass $\left(M_{\mathrm{r}}\right)$ 5000], PEO $\left(M_{\mathrm{r}} 4000000\right)$, tris(hydroxymethyl)aminomethane (THAM), and SDS were from Sigma (St. 
Louis, MO, USA). More details about buffers are shown in the Results and discussion section.

\subsection{Capillary equilibrium and separation}

Capillaries were pre-equilibrated with $0.1 M$ $\mathrm{NaOH}$ overnight before use for electrophoretic separation. Between runs using the commercial $\mathrm{CE}$ system, the capillary was equilibrated with $0.1 \mathrm{M}$ $\mathrm{NaOH}$ via high pressure (689 $476 \mathrm{~Pa}$ ) for $4 \mathrm{~min}$, and subsequently washed out remaining base with running buffers in the absence of PEO via high pressure for $20 \mathrm{~s}$. Between runs using the laboratory-made CE system with LIF, the capillary was equilibrated with $0.1 M \mathrm{NaOH}$ at $1 \mathrm{kV}$ for $10 \mathrm{~min}$. Subsequently, the remaining base was washed out with running buffers by pressure injection for $1 \mathrm{~min}$. Electrokinetic injections at $4 \mathrm{kV}$ for $2 \mathrm{~s}$ and hydrodynamic injections at $30 \mathrm{~cm}$ for $3 \mathrm{~s}$ were performed in the commercial $\mathrm{CE}$ system and laboratory-made CE system, respectively. Separations were performed in $0.1 \%$ PEO solution containing different concentrations of methanol, SDS, DS and TPAI.

\section{Results and discussion}

\subsection{Effects of buffer additives}

To minimize adsorption of PAHs on the capillary wall, the separations were performed in the presence of methanol and PEO. After sample injections, $0.1 \%$ PEO solution (neutral) entered the capillaries by EOF. With a greater migration mobility (equal to EOF mobility) toward the cathode, PEO (in the anodic vial) traversed sample zones that are partitioned in SDS pseudostationary phase during separation. Because of increases in viscosity and slight adsorption of PEO on the capillary wall, bulk EOF mobility gradually decreased during separation. Although bulk EOF slightly decreased during separation, reproducibility was not an issue, which was supported by the fact that the relative standard deviation (RSD) value for bulk EOF mobility was reduced from $3.5 \%$ to less than $1.0 \%$ in presence of $0.1 \%$ PEO and $50 \%$ methanol. This indicates that adsorption of PAHs decreased in the presence of PEO and methanol and the capillary wall was refreshed after treatment with $0.1 M \mathrm{NaOH}[28,29]$. The electrophoretic mobility of PAH-SDS micelle complexes decreased in PEO, leading to changes in the $k^{\prime}$ for PAHs. As a result, differential migration times between any two adjacent peaks were changed, which in turn led to variations of resolution. Decreases in diffusion and adsorption of PAHs on the capillary wall also contributed to changes in resolution. For example, naphthalene and azulene, and 2,3-benzofluorene and fluoranthene were further separated apart and the peak corresponding to benzo $[a]$ pyrene was comparatively sharper in the presence of PEO. Although resolution may be further optimized using high concentrations of PEO, a greater baseline shift and small bulk EOF are problematic.

To conveniently evaluate effects of methanol and SDS on separation of PAHs, the differential migration times between the most hydrophilic analytes (mesityl oxide) and hydrophobic analytes (benzo[a]pyrene) among our selected compounds vs. concentration of methanol and SDS were plotted in Fig. 1A and B, respectively. Because of these two compounds are highly hydrophilic and hydrophobic, the magnitude of the differential migration time should be very close to the width of the separation window $\left(t_{\mathrm{mc}}-t_{0}\right.$, in which $t_{\mathrm{mc}}$ and $t_{0}$ are the migration time for micelles and the analyte that are not partitioned in micelles), which is commonly used to evaluate selectivity in MEKC. At a constant concentration of SDS $(50 \mathrm{mM})$ and DS $(0.02 \%)$, the separation window increased dramatically when using PEO solutions containing less than 50\% methanol (Fig. 1A). At methanol concentrations higher than $50 \%$, resolution was poor, presumably because SDS did not form micelles at high methanol concentrations. In other words, partial separation is most likely due to interactions with SDS monomers and/or DS. When using buffers containing less than $40 \%$ methanol, not only were separations slow and irreproducible, but also the peak corresponding to benzo $[a]$ pyrene was very broad. It is also interesting to note that, with increasing methanol concentrations, mesityl oxide migrated more closely to the interface (caused an indirect peak) between PEO and buffers filled in the capillary prior to analysis. At $60 \%$ methanol, the indirect peak disappeared, which supported our suggestion that $50 \mathrm{~m} M$ SDS did not form 


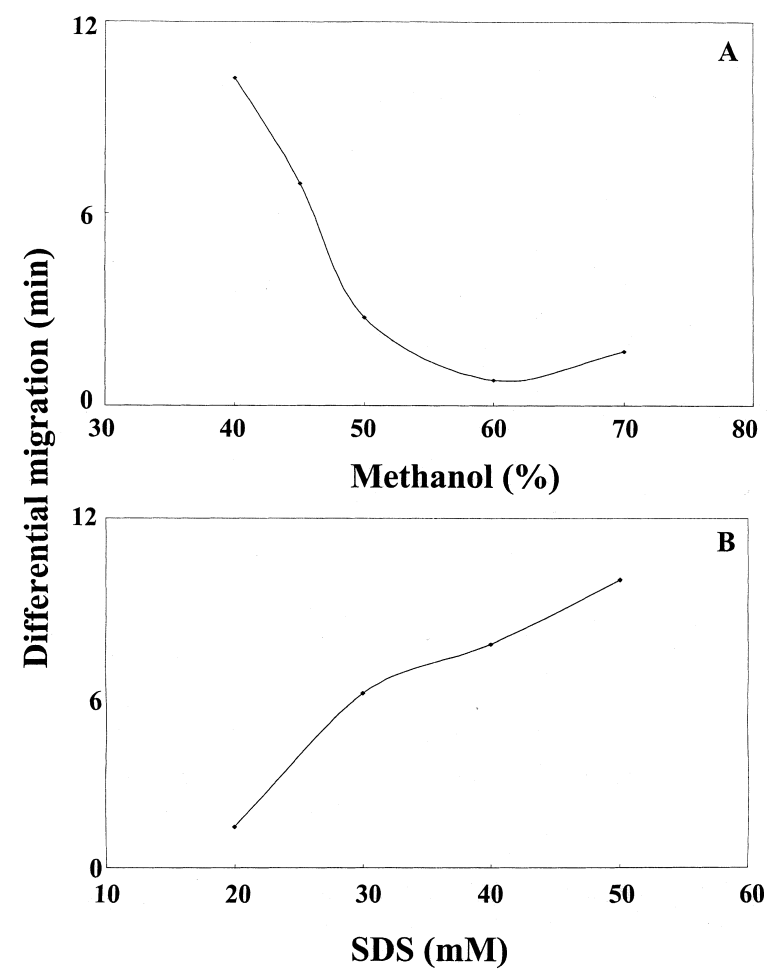

Fig. 1. Effects of methanol and SDS on the separation of five selected PAHs at $15 \mathrm{kV}$. (A) Effect of methanol in a buffer containing $5 \mathrm{~m} M$ THAM, $0.1 \%$ PEO, $50 \mathrm{~m} M$ SDS, and $0.02 \%$ DS; (B) effect of SDS in a buffer containing $5 \mathrm{~m} M$ THAM, $0.1 \%$ PEO, 50\% methanol, and $0.02 \%$ DS. Capillary: $35 \mathrm{~cm}(30 \mathrm{~cm}$ effective length), filled with buffers as in A and B without PEO, respectively. Migration windows are the differential migration times between benzo[ $a]$ pyrene and mesityl oxides.

micelles in $60 \%$ methanol. Thus, the migration time of mesityl oxide was used to calculate EOF mobility and the result was $2.59 \cdot 10^{-4} \mathrm{~cm}^{2} \mathrm{~V}^{-1} \mathrm{~s}^{-1}(\mathrm{RSD}<$ $1.0 \%)$. In an attempt to find a suitable SDS concentration, we performed separation of PAHs in $0.1 \%$ PEO solution containing 50\% methanol, $0.02 \%$ DS and SDS. As predicted, separation windows increased with increasing the SDS concentration over 20-50 $\mathrm{m} M$ (Fig. 1B). At high SDS concentrations $(>60 \mathrm{mM})$, the last peak disappeared; the last second peak became much broader; and reproducibility declined. In terms of resolution, speed, and reproducibility, the optimum SDS concentration is $50 \mathrm{~m} M$.

\subsection{Effect of TPAI and DS}

In order to clarify the effect of DS (polysaccharide containing sulfate) on separation of PAHs, we performed separations in $0.1 \%$ PEO solution containing $50 \mathrm{~m} M$ SDS, $50 \%$ methanol, and DS with concentrations ranging from 0.00 to $0.03 \%$. Over this range, the migration times for benzo[a]pyrene changed from 17.1 to $10.1 \mathrm{~min}$. Fig. 2A shows that the differential time between mesityl oxide and benzo $[a]$ pyrene decreased with increasing DS. This is because DS, with a small electrophoretic mobility (EPM), competed with SDS micelles to form complexes with PAHs. The decreasing trend is in good agreement with the suggestion that glucose effective-
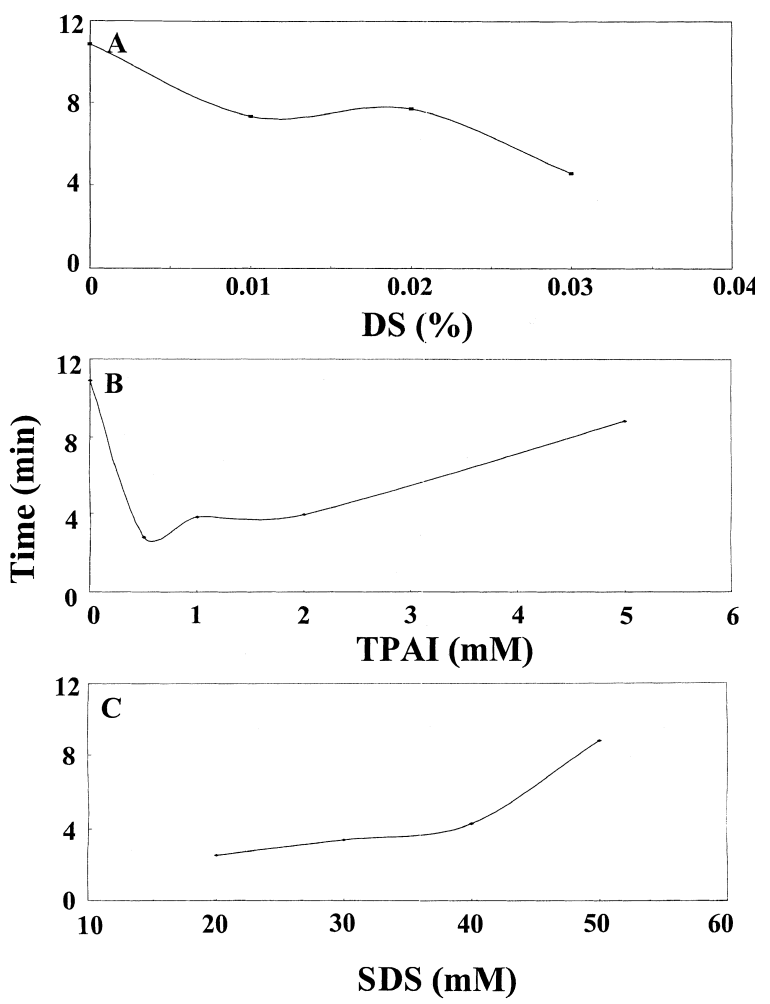

Fig. 2. Effects of additives on the separation of five selected PAHs at $15 \mathrm{kV}$. (A) Effect of DS in a buffer containing $5 \mathrm{mM}$ THAM, 0.1\% PEO, 50\% methanol, and $50 \mathrm{~m} M$ SDS. (B) Effect of TPAI in a buffer containing $5 \mathrm{mM}$ THAM, $0.1 \%$ PEO, $50 \%$ methanol, and $50 \mathrm{~m} M$ SDS. (C) Effect of SDS in a buffer containing $5 \mathrm{~m} M$ THAM, $0.1 \%$ PEO, $50 \%$ methanol, and $5 \mathrm{mM}$ TPAI. Other conditions as in Fig. 1. 
ly decreases the capacity factors of PAHs in the SDS pseudostationary phase [30]. Further increases in DS, resolution lost due to too weak interaction with SDS micelles and Joule heating. In terms of speed and resolution, the optimum concentration of DS is about $0.02 \%$.

Compared to complexes formed with DS (anion), complexes formed between PAHs and cationic TPAI should migrate faster toward the cathode end. As a consequence, separation time should be shorter in the presence of TPAI. To evaluate effects of TPAI on separation of PAHs, we performed separations in $0.1 \%$ PEO solution containing $50 \mathrm{~m} M$ SDS and $50 \%$ methanol and TPAI with concentrations ranging from 0 to $5 \mathrm{mM}$. Over this range, the migration times for benzo[ $a]$ pyrene changed from 17.1 to $14.9 \mathrm{~min}$. The change was less than that in presence of DS. To clearly show effect of TPAI on selectivity, the differential migration times between mesityl oxide and benzo $[a]$ pyrene were depicted in Fig. 2B. It shows the differential migration time decreased with increases in TPAI concentrations below $0.5 \mathrm{mM}$, while it increased over $0.5-5 \mathrm{~m} M$. This is because bulk EOF decreased due to adsorption of TPAI on the capillary wall, which was profound at high concentrations of TPAI. In order to see the role of TPAI in determining resolution, we varied SDS concentrations from 10 to $50 \mathrm{~m} M$ at $5 \mathrm{~m} M$ TPAI. At a constant TPAI concentration, the differential migration times between mesityl oxide and benzo $[a]$ pyrene increased dramatically with increasing SDS concentrations above $40 \mathrm{~m} M$ (Fig. 2C). This is probably SDS did not form stable micelles below 40 $\mathrm{m} M$ in $50 \%$ methanol. As a consequence, TPAI competed strongly with SDS monomers to form complexes with PAHs.

To further compare effects of DS and TPAI on the separation of PAHs in MEKC, the separations of PAHs in the absence of TPAI and DS, in the presence of $0.03 \% \mathrm{DS}$, and $1 \mathrm{~m} M$ TPAI, respectively, were shown in Fig. 3A-C. The separation time was shortened either in the presence of DS or TPAI, which supported our above-mentioned suggestions that DS or TPAI competed with SDS to form complexes with PAHs. In order to elucidate the role of TPAI or DS in determining separation, resolution values between any two adjacent peaks were plotted in Fig. 4. Although resolution was slightly lost in the

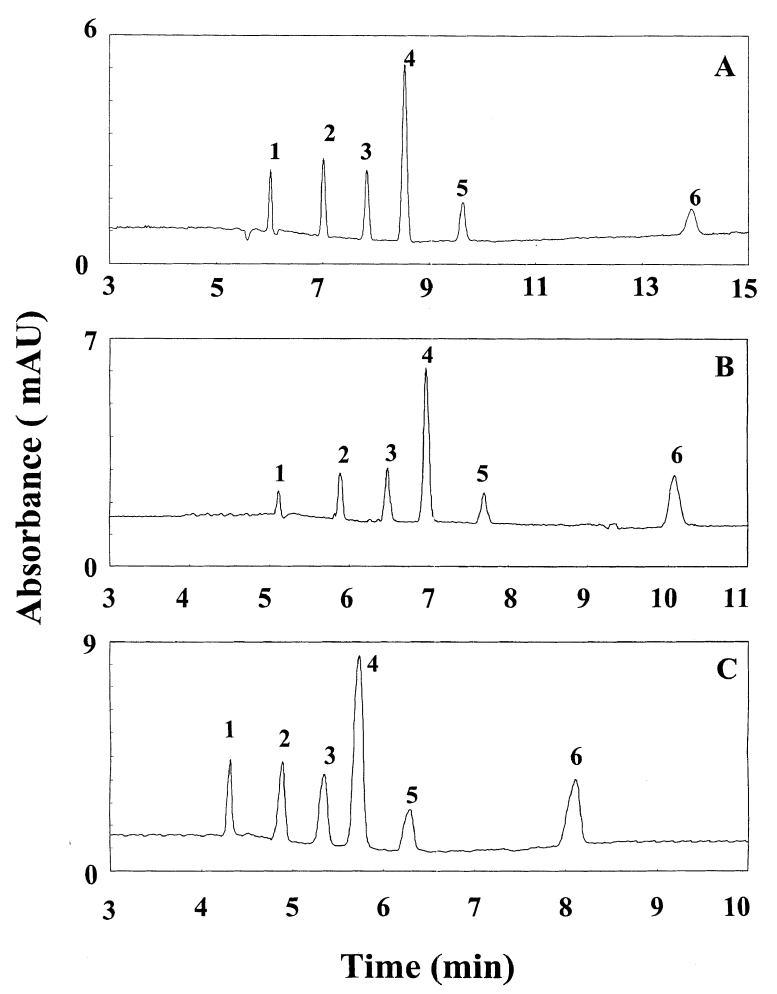

Fig. 3. Separations of five selected PAHs in $0.1 \%$ PEO solution containing $5 \mathrm{~m} M$ THAM, $50 \%$ methanol, and $50 \mathrm{~m} M$ SDS at 15 kV. (A) No TPAI and DS; (B) $0.03 \%$ DS; (C) $1 \mathrm{~m} M$ TPAI. Peak identities: $1=$ mesityl oxide; $2=$ naphthalene; $3=$ fluorene; $4=$ anthracene; $5=$ pyrene; and $6=$ benzo $[a]$ pyrene. Other conditions as in Fig. 1.

presence of either DS or TPAI, separation was faster and reproducibility was improved (the RSD values for migration time of benzo $[a]$ pyrene were 2.5 and $5.0 \%$ in the presence and absence of DS, respectively). Overall, in terms of speed and resolution, a buffer containing DS is more suitable for separation of PAHs.

\subsection{Separation of PAHs}

Ten selected PAHs with ring sizes 2 to 5 shown in Fig. 5 were separated in $0.1 \%$ PEO solution either containing $50 \mathrm{~m} M$ SDS, $0.02 \%$ DS, and $45 \%$ or $50 \%$ methanol. Fig. 6A shows these analytes were separated in less than $12 \mathrm{~min}$, while the peak corresponding to benzo $[a]$ pyrene was quite broad when 


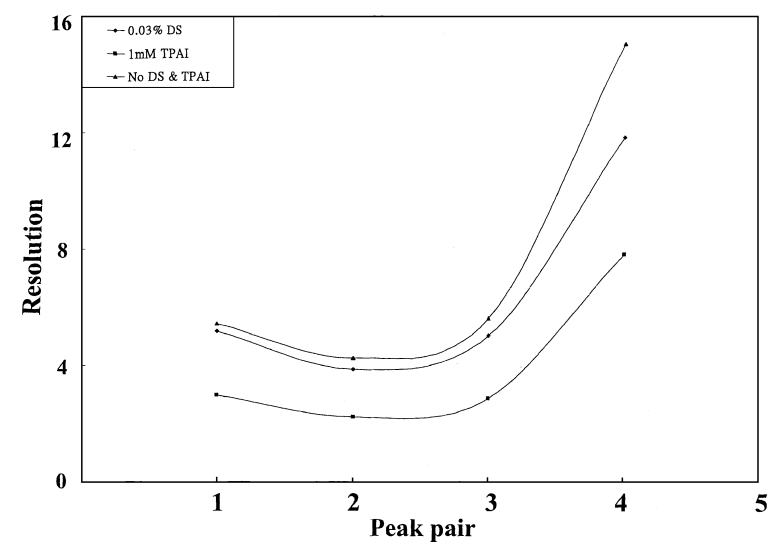

Fig. 4. Comparison of effects of DS and TPAI on resolution of separating five selected PAHs. Resolution values were calculated from data obtained from Figs. 3A-C. Peak pairs: 1=mesityl oxide and naphthalene, $2=$ naphthalene and fluorene, $3=$ fluorene and anthracene, $4=$ anthracene and pyrene, and $5=$ pyrene and benzo[a]pyrene.

using buffers containing $45 \%$ methanol. Although the peak corresponding to benzo $[a]$ pyrene became very sharp and more symmetric, 7,8-benzoquinoline and biphenyl were not resolved, and naphthalene and azulene were only partially resolved when using buffers containing 50\% methanol (Fig. 6B). The migration order shown in both electropherograms corresponds to the number of benzene rings (hydrophobicity), except that for 7,8-benzoquinoline. At low methanol concentrations (45\%), adsorption of PAHs was more serious. As a result, peaks were broader and separation was less reproducible (the RSD values of the migration time for benzo $[a]$ pyrene was $3.5 \%$ ).

\subsection{Detection limit}

Adsorption of PAHs on the capillary wall is less when injecting a small amount of samples. To minimize the problems associated with poor solubility and adsorption of PAHs in MEKC, using a sensitive detection system is one of the choices. For this purpose, we performed analysis using a laboratory-made CE system with LIF. Because the maximum excitation wavelengths for PAHs are distributed in a wide range, which are related to the ring size and substituted groups, the quantum yields for the above 10 selected PAHs are not high when excited at $325 \mathrm{~nm}$ using a $\mathrm{He}-\mathrm{Cd}$ laser. In order to test our hypothesis regarding solubility problems, we selected and tested seven PAHs with higher quantum yields at $325 \mathrm{~nm}$. As seen in Table 1, the emission wavelengths for these analytes are also quite different. To simplify our setup, a cut-off filter was used, which allowed more lights reaching the detector. As a result, a higher background and a greater noise were expected, leading to higher limit of detection (LOD). The LOD [signal-to-noise ratio $(S / N)=3$ ] for these PAHs shown in Table 1 are in $\mathrm{n} M$ to tenths of $\mathrm{n} M$ levels, which are about 100-1000-fold improvements in sensitivity compared to those obtained with absorbance detection. The LOD can even be lowered by performing analysis using a laser-induced dispersed fluorescence detection with multiple excitation lines and a multiple detection system [31].

\subsection{Stepwise technique}

As a result of better sensitivity with an LIF detection mode, more diluted samples were injected, which allowed us to use buffers containing low methanol and SDS for the following experiments without causing irreproducible problems. The use of buffers containing less amounts of SDS also benefited for a low fluorescent background and high quantum yields (minimized Joule heating). Fig. 7A shows that the separation of the seven PAHs in $0.1 \%$ PEO solution containing $0.02 \%$ DS, $35 \mathrm{~m} M$ SDS and $40 \%$ methanol was accomplished in $60 \mathrm{~min}$. Further increases in methanol concentrations, separation was faster but resolution was diminished. On the other hand, Fig. 7B shows that seven peaks were detected in $20 \mathrm{~min}$ in $0.1 \%$ PEO solution containing $0.02 \%$ DS, $20 \mathrm{~m} M$ SDS and 50\% methanol. However baseline resolution was not achieved between 7,8benzoquinoline and 2,3-benzofluorene and among anthracene, fluoranthene, and pyrene. Please note that SDS micelles did not form under this condition. Although resolution increased with increasing SDS concentrations, sensitivity was deteriorated due to Joule heating. Thus, to optimize resolution, speed, and sensitivity, a gradient or stepwise technique should be useful. For example, a gradient technique in conjunction with changes in acetonitrile concentration has been developed for this purpose in CEC 
<smiles>c1ccc2ccccc2c1</smiles>

Naphthalene<smiles>c1ccc2c(c1)cc1ccc3cccc4ccc2c1c34</smiles>

Benzo[a]pyrene<smiles>c1ccc2cc3ccccc3cc2c1</smiles>

Anthracene

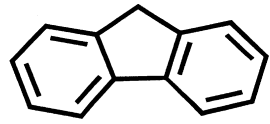

Fluorene

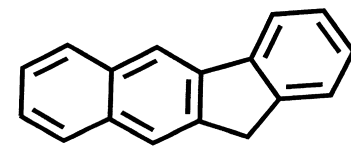

2,3-Benzofluorene

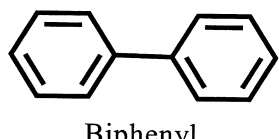

Biphenyl<smiles>c1ccc2cccc-2cc1</smiles>

Azulene

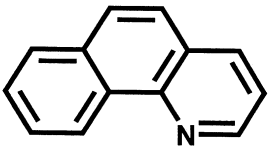

7.8-Benzoquinoline<smiles></smiles>

Pyrene

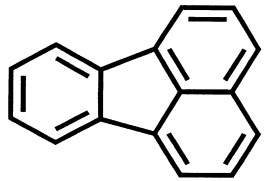

Fluoranthene

Fig. 5. Structures of 10 selected PAHs used in analysis with a commercial CE system.

[32]. Because SDS micelles with a greater electrophoretic mobility, while methanol with a smaller EPM than PAHs complexes toward the anode, separations were performed in $0.1 \%$ PEO solution containing $35 \mathrm{~m} M$ SDS and $40 \%$ methanol at $15 \mathrm{kV}$ for $120 \mathrm{~s}$, and subsequently in $0.1 \%$ PEO solution containing $20 \mathrm{~m} M$ SDS and $50 \%$ methanol for the rest. Under this stepwise condition, PAHs traversed both zones containing SDS micelles and 50\% methanol. As a consequence, resolution, sensitivity and reproducibility should be optimized. Fig. 7C shows that the separation with reasonable resolution was complete in $30 \mathrm{~min}$. The RSD values for migration times of benzo $[a]$ pyrene shown in these three electropherograms were $4.5,2.0$, and $2.5 \%$, respectively.

\section{Conclusions}

We performed the separation of PAHs in $0.1 \%$ PEO solution containing SDS, methanol, and additives. In the presence of PEO, bulk EOF was regulated and adsorption of PAHs on the capillary wall was reduced, leading to reproducibility and optimum resolution. In addition, DS was added to the buffers for optimum speed, resolution, and reproducibility. The separation of 10 PAHs was accomplished less than 12 min in $0.1 \%$ PEO solution containing $50 \mathrm{~m} M$ SDS, $0.02 \%$ DS, and $45 \%$ methanol. Resolution, sensitivity, speed, and reproducibility were also optimized by a stepwise technique associated with changes in SDS and methanol 


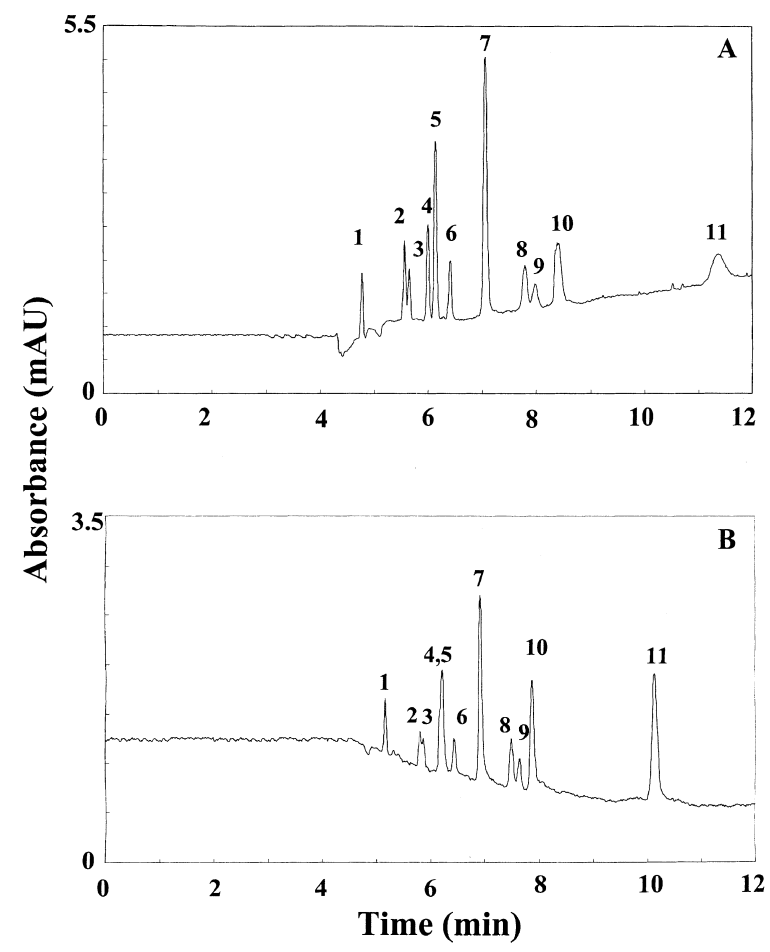

Fig. 6. Separation of 10 selected PAHs in $0.1 \%$ PEO solution containing $5 \mathrm{~m} M$ THAM, $50 \mathrm{~m} M$ SDS, $0.02 \%$ DS and $45 \%$ methanol (A) or $50 \%$ methanol (B) at $15 \mathrm{kV}$. Peak identities: $1=$ mesityl oxide; 2 =naphthalene; $3=$ azulene; $4=7,8$-benzoquinoline; $5=$ biphenyl; $6=$ fluorene; $7=$ anthracene; $8=2,3$-benzofluorene; $9=$ fluoranthene; $10=$ pyrene; and $11=$ benzo $[a]$ pyrene. The concentrations of analytes are: $2 \cdot 10^{-3} M$ mesityl oxide, 2.67 $\cdot 10^{-3} M$ naphthalene, $1.33 \cdot 10^{-3} M$ biphenyl, $6.67 \cdot 10^{-4} M$ azulene, $2 \cdot 10^{-4} M$ benzo $[a]$ pyrene, anthracene, and $3.33 \cdot 10^{-4} M$ 7,8-benzoquinoline, 7,8-benzoquinoline, 2,3-benzofluorene, pyrene, fluoranthene and fluorene. Other conditions as in Fig. 1.

Table 1

LOD values for PAHs using a $\mathrm{He}-\mathrm{Cd}$ laser at $325 \mathrm{~nm}$ in $\mathrm{MEKC}^{\mathrm{a}}$

\begin{tabular}{llll}
\hline PAH & $\lambda_{\text {ex }}(\mathrm{nm})$ & $\lambda_{\text {em }}(\mathrm{nm})$ & $\mathrm{LOD}^{\mathrm{b}}(\mathrm{n} M)$ \\
\hline 3-Aminofluoroanthene & 305 & 480 & 14.7 \\
7,8-Benzoquinoline & 300 & 347 & 2.64 \\
2,3-Benzofluorene & 300 & 500 & 3.13 \\
Anthracene & 305 & 405 & 1.80 \\
Fluoranthene & 305 & 480 & 2.40 \\
Pyrene & 295 & 390 & 1.20 \\
Benzo[ $a$ ]pyrene & 305 & 410 & 4.85 \\
\hline
\end{tabular}

\footnotetext{
${ }^{\mathrm{a}}$ Separation in $0.1 \%$ PEO solution containing $20 \mathrm{~m} M$ SDS, $0.02 \%$ DS, and $50 \%$ methanol using a capillary filled with buffers containing $35 \mathrm{mM}$ SDS, $40 \%$ methanol, and $0.02 \%$ DS.

${ }^{\mathrm{b}}$ Signal-to-noise ratio $=3$.
}

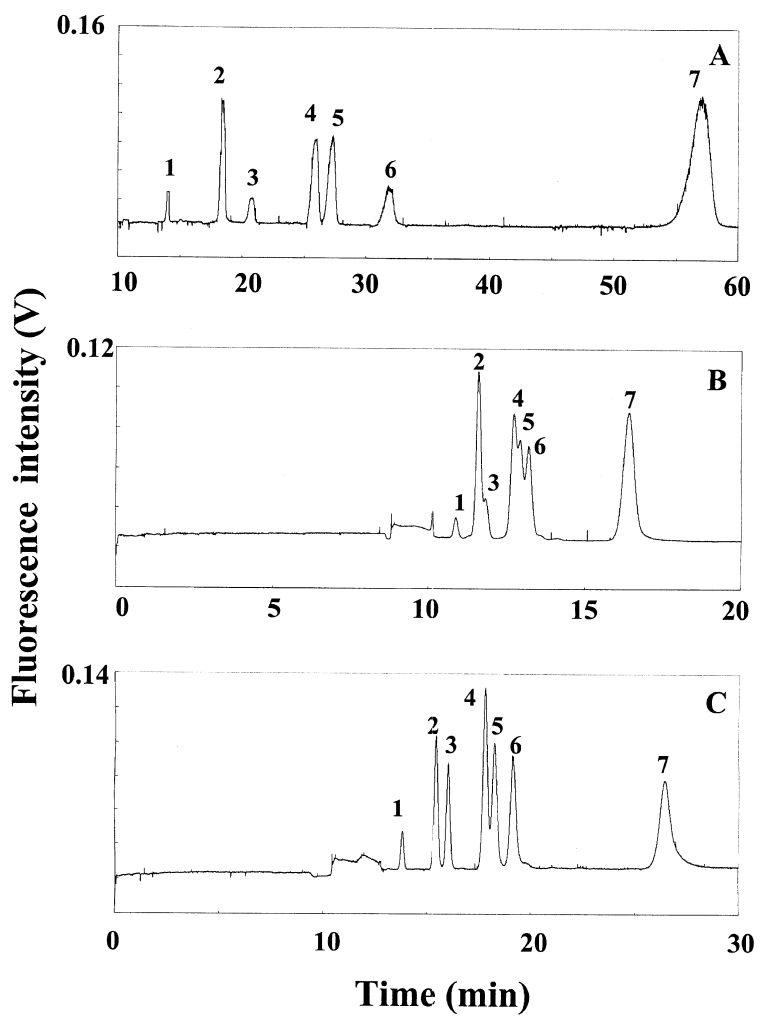

Fig. 7. Separations of PAHs under isocratic conditions and under stepwise changes in SDS and methanol concentrations, respectively. (A) Separation was performed in $0.1 \%$ PEO solution containing $5 \mathrm{~m} M$ THAM, $35 \mathrm{~m} M$ SDS, $0.02 \%$ DS and $40 \%$ methanol at $15 \mathrm{kV}$. (B) Separation was performed in $0.1 \%$ PEO solution containing $5 \mathrm{~m} M$ THAM, $20 \mathrm{~m} M$ SDS, $0.02 \%$ DS and $50 \%$ methanol at $15 \mathrm{kV}$. (C) Separation was performed in $0.1 \%$ PEO solution containing $5 \mathrm{~m} M$ THAM, $35 \mathrm{~m} M$ SDS and $40 \%$ methanol at $15 \mathrm{kV}$ for $120 \mathrm{~s}$, subsequently in $0.1 \%$ PEO solution containing $5 \mathrm{~m} M$ THAM, $20 \mathrm{~m} M$ SDS and $50 \%$ methanol for the rest. Peak identities: 1=3-aminofluoranthene $(1 \mu M)$; 2=7,8benzopquinoline $(2 \mu M)$; 3=2,3-benzofluorene $(1 \mu M) ; 4=$ anthracene $(1 \mu M) ; 5=$ fluoranthene $(1 \mu M) ; 6=$ pyrene $(0.5 \mu$ $M) ; 7=$ benzo $[a]$ pyrene $(1 \mu M)$. Capillary: $50 \mathrm{~cm}(40 \mathrm{~cm}$ effective length); and filled with buffers containing $5 \mathrm{mM}$ THAM, 35 $\mathrm{m} M$ SDS, $0.02 \%$ DS and $40 \%$ methanol.

concentrations. It is suggested that buffers containing a high concentration of SDS and a low percentage of methanol are used for selectivity, while buffers containing low concentrations of SDS and a higher percentage of methanol are performed later for detection and speed. The success of this stepwise technique for separating highly hydrophobic neutral 
compounds indicated $\mathrm{CE}$ should be useful for monitoring environmental and biological samples.

\section{Acknowledgements}

This work was supported by a grant from Chinese Petroleum Chemical Cooperation and a grant from National Taiwan University.

\section{References}

[1] S. Terabe, K. Otsuka, K. Ichikawa, A. Tsuchiya, T. Ando, Anal. Chem. 56 (1984) 111.

[2] A. Zemann, D. Volgger, Anal. Chem. 69 (1997) 3243.

[3] R.M. Seifar, J.C. Kraak, W.Th. Kok, Anal. Chem. 69 (1997) 2772.

[4] W. Ding, J.S. Fritz, Anal. Chem. 70 (1998) 1859.

[5] Z.E. Rassi, J. Chromatogr. A 875 (2000) 207.

[6] T. Vo-Dinh (Ed.), Chemical Analysis of Polycyclic Aromatic Compounds, Wiley, New York, 1989.

[7] G. Xue, H.-M. Pang, E.S. Yeung, Anal. Chem. 71 (1999) 2642.

[8] X. Xu, R.J. Hurtubise, J. Chromatogr. A 829 (1999) 289.

[9] A.-L. Nguyen, J.H.T. Luong, Anal. Chem. 69 (1997) 1726.

[10] W. Ding, J.S. Fritz, Anal. Chem. 69 (1997) 1593.

[11] C. Fujimoto, Y. Fujise, E. Matsuzawa, Anal. Chem. 68 (1996) 2753.

[12] S. Terabe, Trends Anal. Chem. 8 (1984) 129.

[13] Y. Mechref, Z.E. Rassi, Anal. Chem. 68 (1996) 1771.

[14] H. Nishi, M. Matsuo, J. Liq. Chromatogr. 14 (1991) 973.
[15] Z. Liu, H. Zou, M. Ye, J. Ni, Y. Zhang, J. Chromatogr. A 863 (1999) 69.

[16] C. Yan, R. Dadoo, R.N. Zare, D.J. Rakestraw, D.S. Anex, Anal. Chem. 68 (1996) 2726.

[17] P.G. Muijselaar, H.B. Verhelst, H.A. Claessens, C.A. Cramers, J. Chromatogr. A 764 (1997) 323.

[18] J.L. Miller, M.G. Khaledi, D. Shea, Anal. Chem. 69 (1997) 1223.

[19] S. Yang, J.G. Bumgarner, M.G. Khaledi, J. High. Resolut. Chromatogr. 18 (1995) 443.

[20] C.P. Palmer, S. Terabe, Anal. Chem. 69 (1997) 1852.

[21] S.A. Shamsi, C. Akbay, I.M. Warner, Anal. Chem. 70 (1998) 3078.

[22] J.-L. Liao, N. Chen, C. Ericson, S. Hjerten, Anal. Chem. 68 (1996) 3468

[23] P.B. Wright, A.S. Lister, J.G. Dorsey, Anal. Chem. 69 (1997) 3251.

[24] G.W. Sovocool, W.C. Brumley, J.R. Donnelly, Electrophoresis 20 (1999) 3297.

[25] C. Yan, R. Dadoo, H. Zhao, R.N. Zare, D.J. Rakestraw, Anal. Chem. 67 (1995) 2026.

[26] R.S. Brown, J.H.T. Luong, O.H.J. Szolar, A. Halasz, J. Hawari, Anal. Chem. 68 (1996) 287.

[27] R. Dadoo, R.N. Zare, C. Yan, D.S. Anex, Anal. Chem. 70 (1998) 4787.

[28] H.-S. Chen, H.-T. Chang, Anal. Chem. 71 (1999) 2033.

[29] H.-T. Chang, H.-S. Chen, M.-M. Hsieh, W.-L. Tseng, Rev. Anal. Chem. 19 (2000) 1.

[30] T. Kaneta, S. Tanaka, M. Taga, H. Yoshida, J. Chromatogr. 609 (1992) 369.

[31] M.G. Garguilo, D.H. Thomas, D.S. Anex, D.J. Rakestraw, J. Chromatogr. A 883 (2000) 231.

[32] C. Yan, R. Dadoo, R.N. Zare, D.J. Rakestraw, D.S. Anex, Anal. Chem. 68 (1996) 2726. 